\title{
Análisis crítico del discurso al campo del currículum de la formación inicial docente en Chile
}

\author{
Critical discourse analysis to the field of curriculum of initial teacher training in Chile \\ Análise crítica do discurso no campo do currículo da formação inicial \\ de professores no Chile
}

\author{
Héctor Gómez Cuevas. ${ }^{a}$ \\ a Universidad Católica Silva Henríquez, Santiago, Chile. Fono: 56-27950573. \\ Correo electrónico: hgomez@ucsh.cl
}

\begin{abstract}
RESUMEN
El presente artículo expone el ejercicio epistemológico de levantar un esquema analítico para problematizar, desde una perspectiva postestructural, el discurso del currículum de formación inicial docente en Chile, a partir del análisis crítico del discurso. Las reflexiones que se exponen dan cuenta de la complejidad en tender puentes de diálogo entre un tipo de análisis inserto en un paradigma estructural, considerando miradas postestructurales del conocimiento. De este modo, la argumentación principal se desarrolla en torno a la generación de caminos investigativos abiertos a la incertidumbre, en un contexto de crítica a la pretensión objetiva de la cientificidad en Ciencias Sociales.
\end{abstract}

Palabras clave: currículum, formación inicial docente, análisis crítico del discurso, perspectiva postestructural.

\begin{abstract}
This article exposes the epistemological exercise to generate an analytical framework to problematize, from a poststructural perspective, the discourse of initial teacher training curriculum in Chile through a critical discourse analysis. The reflections are presented, showing the complexity of building bridges of dialogue between a kind of self-analysis of a structural paradigm, considering poststructural viewpoints of knowledge. Thus, the main argument revolves around the generation of research trails open to uncertainty within critique context of the scientific objective claim of Social Sciences.
\end{abstract}

Key words: curriculum, initial teacher training, critical discourse analysis, poststructural perspective.

\section{RESUMO}

Expõe o esforço epistemológico para levantar um esquema analítico para problematizar, desde uma perspectiva pós-estruturalista, o discurso do currículo da formação inicial de professores no Chile, a partir de análise crítica do discurso. As reflexões que são expostas dão conta da complexidade da construção de pontes de diálogo entre um tipo de análise inserida em um paradigma estrutural, considerando o enfoque pós-estruturalista do conhecimento. Assim, o principal argumento se desenvolve em torno da geração de caminhos investigativos abertos à incerteza no contexto de crítica à pretensão objetiva da cientificidade em Ciências Sociais.

Palavras chave: currículo, formação inicial de professores, análise crítica do discurso, perspectiva pósestruturalista. 
La clásica concepción del método al servicio de la teoría como instrumento para establecer la generalidad no representa el espíritu de este estudio. Por una parte, desde una perspectiva que apunta a la complejidad y a la riqueza del proceso investigativo, la construcción de un discurso metodológico es concebida, en términos de Foucault (2008), como una caja de herramientas, entendida como los insumos que dialogan y permiten dar riqueza a un estudio. Por otra parte, en términos de Fairclough (2003), resulta fundamental la comprensión de la metodología de la investigación, como un diálogo teoría-práctica, que el autor denomina 'teorético', permite formas complementarias de leer un evento social complejo.

Esta concepción teorética orienta la elección del Análisis Crítico del Discurso (ACD) como una teoría y un método, "una perspectiva teorética que versa sobre el lenguaje, y en un sentido más general, sobre la semiosis que se encuentra en relación dialógica con otras teorías y métodos sociales" (Fairclough, 2003: 179-180). Dicha elección da cuenta de un proceso reflexivo, crítico y postcrítico de adaptación de la 'caja de herramientas', propio de las investigaciones de enfoque cualitativo, en la medida que las motivaciones del investigador hacia la producción de saberes no se orientan a la generalidad y la réplica, ni persiguen fundamentarse en la medición y en la estadística, sino en la profundización y problematización ${ }^{2}$ de los análisis.

Este estudio adhiere, por tanto, al enfoque cualitativo, en la medida que asume un proceso inductivo, recurrente, analizando la realidad con énfasis en los procesos subjetivos y la no secuencia lineal. Asimismo, se fundamenta en la necesidad de profundizar en las ideas, dar amplitud, reforzar y valorar lo interpretativo y la contextualización del fenómeno de estudio. Así, en función de lo descrito anteriormente, la metodología propuesta descansa sobre tres pilares o principios.

En primer lugar, la importancia del lenguaje en la interacción social, por cuanto en él quedan trazadas las relaciones de poder, que han establecido una serie de reglas y pautas que regulan su uso (Foucault, 1970). Bajo esta concepción, lo que decimos y hacemos a través del lenguaje forma parte de una práctica discursiva asumida mediante modelos y pautas de acción, originada en las convicciones y los valores compartidos por una sociedad en un momento histórico determinado (Cherryholmes, 1999). Asimismo, Butler señala que "si estamos formados por el lenguaje, entonces ese poder constitutivo procede y condiciona cualquier decisión que pudiéramos tomar sobre él, insultándonos desde el principio, desde su poder previo" (1997: 16).

En segundo lugar, entender el corpus, en este caso, el currículum de formación de profesores/as, como un discurso, equivale a abordarlo como "una práctica social, (...) una forma de acción entre las personas que se articula a partir del uso lingüístico contextualizado, ya sea oral o escrito. El discurso es parte de la vida social y a la vez un instrumento que crea vida social" (Fairclough, 1989: 28). Cabe señalar que este discurso, según Foucault en sus obras El orden del discurso (1970) y La arqueología del saber (1989), posee reglas que regulan su uso, convirtiéndose el discurso en un conjunto de reglas

Es decir, incorpora elementos teóricos y metodológicos de forma articulada, no asumiendo una separación entre ellos.

Se emplea la acción de 'problematizar', según lo señalado por Íñiguez, en tanto "refiere a la totalidad de prácticas discursivas y no discursivas que introduce algo en el juego de lo verdadero y de lo falso y lo constituye como un objeto de pensamiento...", y que "la problematización pone en duda todo aquello que se da por evidente o por bueno, cuestiona lo que está constituido como incuestionable, recela de aquello que es indudable" (2003: 78-79). 
anónimas, históricas y contextuales, que regulan sus condiciones de funcionamiento en un ámbito social específico. Adicionalmente, los discursos han de ser tratados como prácticas que sistemáticamente constituyen los objetos de los que hablan.

En tercer lugar, destaca la necesidad de estudiar el discurso desde distintas disciplinas, propósitos y métodos diferentes en base a una multidisciplinariedad. Por una parte, como señala Meyer (2003), el ACD adopta elementos teoréticos de muy distinto origen, no existiendo ningún punto de vista teorético guía unánime, y por otra, de acuerdo con Van Dijk (2009), los discursos forman parte de interacciones conversacionales, textos escritos y gestos, mímicas, disposiciones tipográficas, imágenes, entre otros, ante los cuales existen diversas formas de análisis, que enriquecen los estudios y permiten generar diálogos entre teorías y métodos.

Optar por uno u otro paradigma investigativo pareciera estar encapsulado en la binariedad de opciones excluyentes para realizar investigaciones, de acuerdo con las concepciones ontológicas, epistemológicas y de método que se asuman a priori de la realización del proceso investigativo. En este escenario, Delgado (1994) señala que la opción cuantitativo/cualitativo forma parte de una red de oposiciones que no se agota, y se alimenta de visiones dogmáticas, que las han constituido como opciones excluyentes. No obstante, en la actualidad, ya no es raro encontrar investigaciones que han sido capaces de triangular ambas formas de investigación, estableciendo un diálogo entre ellas, o bien, reconociendo que en una u otra decisión se están considerando las ventajas que cada una de ellas faculta en función de las necesidades del estudio, sin la pretensión de superioridad.

De acuerdo con lo anterior, la adopción del enfoque cualitativo como territorio epistemológico y metodológico mayor, responde a las características señaladas por Ruiz (1999), tales como la utilización del lenguaje de los conceptos y las metáforas por sobre los números y las estadísticas; el modo de captar la información no estructurada flexible y desestructurada; el procedimiento más inductivo que deductivo; la orientación no particularista y generalizadora, y más holística y concretizadora, y; la apelación a la profundidad del análisis y a la riqueza interpretativa. Sin embargo, en el presente artículo interesa clarificar que la elección del "método's se nutre de una serie de procesos reflexivos, los cuales se describen a continuación.

En primer término, se encuentra la motivación hacia la temática a investigar y los espacios disponibles que acojan los intereses y la complejidad de ésta. Una segunda aproximación dice relación con el recorrido epistemológico del proceso investigativo, que va abriéndose camino hacia la construcción emergente de una metodología adecuada. Y, en tercer lugar, se nutre de la crítica de los modelos tradicionales de investigación y sus opciones excluyentes, que hacen perder riqueza a la investigación misma, situando a la estructura precedente por sobre las necesidades específicas del proceso investigativo.

Asimismo, esta consideración de investigación cualitativa como campo epistemológico mayor, está inserta dentro de un entendimiento más problematizador de 'lo real', que permite cuestionar cómo 'existe' el ser humano y cómo se puede 'entender/comprender' mejor. A su vez, la elección paradigmática contempla lo planteado por Denzin (2008), respecto de un nuevo diálogo entre paradigmas, más que una oposición radical que niega a

Utilizo la palabra método entre comillas, con el fin de clarificar que no se adhiere a esta consideración utilitaria de un conjunto de pasos para llegar a ciertas conclusiones, sino en un diálogo permanente entre teoría y práctica que nutre y enriquece una forma de construir conocimiento, que a su vez involucra diferentes teorías, enfoques, perspectivas y métodos, los cuales permiten problematizar el campo de estudio. 
otro. Dicha consideración persigue la coherencia con la perspectiva postestructural mayor del estudio, que en su proceso interpretativo valora la diferencia y la incertidumbre, ante determinados "criterios que reproducen cierto tipo de ciencia, una ciencia que silencia muchas voces" (Denzin \& Lincoln, 2005: 10).

Ahora bien, la elección específica del Análisis Crítico del Discurso (ACD), como enfoque teorético inserto en la investigación cualitativa (Denzin \& Lincoln, 2005), surge de la concepción de discurso -en los diferentes ámbitos de la convivencia humana- que operan sobre la base de valores, criterios e intereses normados y reglados que, según Foucault (1970), dan origen a un 'orden del discurso', como un escenario de relaciones de poder en una sociedad, que instituyen posibilidades de identidad en un determinado contexto social.

El uso del ACD, desde una mirada postestructural, no está ajeno a ciertas tensiones debido al componente crítico de este enfoque, que apunta al desvelamiento y la generación de conciencia para la transformación. Este componente crítico tensiona la coherencia metodológica, por cuanto forma parte de un componente estructural propio de la modernidad, cuestionado desde el postestructuralismo. Sin embargo, reconociendo dicha tensión, el diálogo entre el ACD y la perspectiva postestructuralista se produce a partir de aspectos ya señalados, tales como: el énfasis en el lenguaje como una forma de legitimación de las relaciones de poder, las posibilidades de generar metodologías de carácter multidisciplinar que aceptan diferentes enfoques, perspectivas, teorías y métodos en diálogo, y finalmente, el ejercicio del/la investigador/a de exponer sus posicionamientos epistemológicos y políticos en el proceso investigativo.

Figura $N^{\circ} 1$. Diálogo entre Análisis Crítico del Discurso y Perspectiva Postestructural

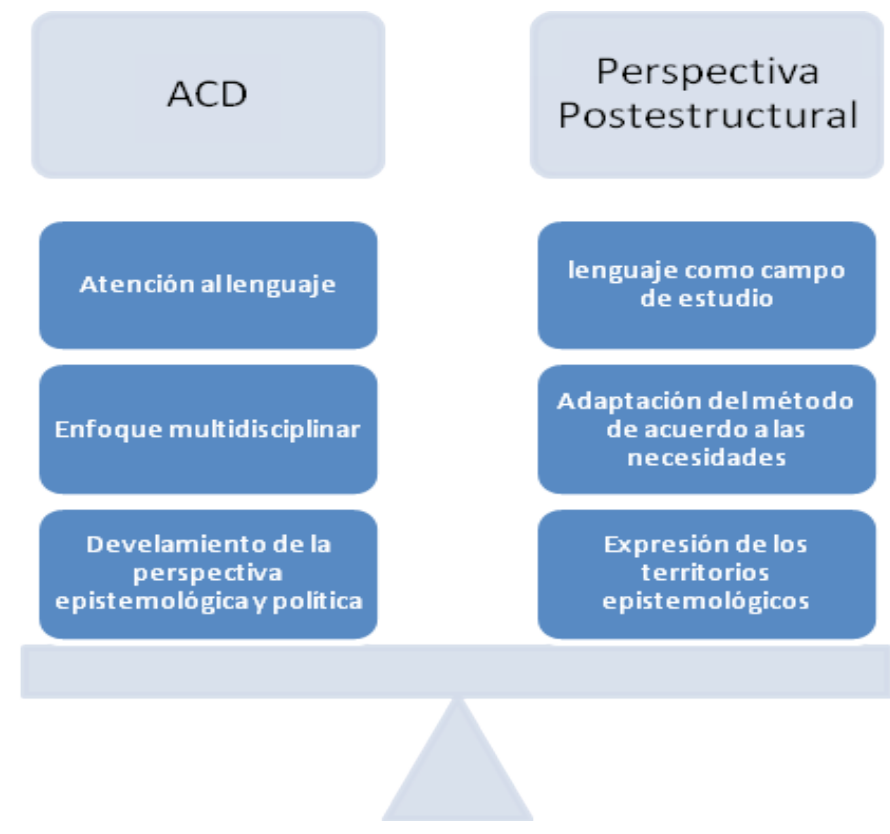

Fuente: elaboración propia. 
En síntesis, el enfoque cualitativo brinda el territorio teórico-metodológico que permite el recorrido del ACD, con la salvedad de reconocer que la elección del enfoque implica una adaptación del método a partir de las necesidades del fenómeno de estudio y el corpus discursivo, y a las características propias de la investigación cualitativa (Denzin \& Lincoln, 2005), y del propio ACD (Fairclough, 2003), por sobre la lógica -exclusiva- binaria que obliga a optar por un enfoque en base a una superioridad epistemológica. En este sentido, hacer análisis crítico del discurso, desde una mirada postestructuralista, involucra asumir el ACD como un tipo de análisis realizado desde una crítica a la ciencia "como disciplina neutra y limpia de las concepciones ideológicas sobre la realidad, -concebida como- forma de saber, sexuada, androcentrista y eurocéntrica" (Palacios, 2009: 66).

Un interesante artículo de Antonio Stecher (2009) resume las diferentes perspectivas del modelo de ACD de Norman Fairclough y sus implicancias metodológicas. En él destaca sus posibilidades para trabajar con subjetividades, experiencias y significados, incorporar perspectivas sociohistóricas, y las posibilidades de complementar o reemplazar las matrices epistemológicas, marcos conceptuales y estrategias metodológicas clásicas, apuntando a perspectivas hermenéuticas, críticas y postestructuralistas, con un fuerte componente histórico y contextual.

Por su parte, Fairclough (2003) señala que el ACD corresponde, por un lado, a una teoría y un método (teorética) que versa sobre el lenguaje y la semiosis, y por otro, a un momento del proceso social material que da origen a formas de analizar el lenguaje o la semiosis, insertos en otros análisis de procesos sociales de carácter más general. La elección del modelo de Fairclough corresponde a una adaptación del mismo, en concordancia con la flexibilidad de la investigación cualitativa, el ACD y las necesidades del estudio.

En términos específicos, se plantea la utilización del Modelo Tridimensional de Análisis Crítico del Discurso propuesto por Norman Fairclough (2009, 2008, 2003, 1995, 1992, 1989), que plantea analizar el discurso considerando tres niveles. El primero de ellos denominado discurso como 'texto' (o práctica textual) corresponde a una concepción del discurso como unidad lingüística o 'pieza de lenguaje' escrito o hablado; un segundo nivel llamado 'práctica discursiva' involucra procesos de producción e interpretación de los textos, y; un tercer nivel descrito como 'práctica social' atañe al carácter situacional e institucional del evento discursivo.

Estos tres niveles no funcionan de forma aislada, sino que se relacionan de forma ascendente, es decir, el nivel de práctica social no puede ser entendido sin considerar el nivel de la práctica y el texto, y de igual modo, el de práctica no puede concebirse sin el texto, donde el discurso como texto es contenido en la práctica, y a su vez, ambos se incorporan dentro de la práctica social. Este modelo tridimensional se utiliza como trama analítica del corpus, con el fin de problematizar los discursos del diseño curricular de la formación de profesores, desde la construcción lingüística específica (texto), los diferentes discursos que dialogan en dicha construcción (práctica), y los elementos de producción, recepción y distribución a nivel social e institucional (práctica social).

De este modo, el discurso como texto se entiende como una pieza del discurso o unidad lingüística (escrito o hablado) y como un producto social. En este nivel, los textos suponen una particular y situada utilización del lenguaje y sus múltiples recursos, a partir de los cuales se actualiza el poder constructivo del discurso, mediante la interacción de sus dimensiones psicológicas, sociales e históricas. Esta consideración analítica conlleva reconocer al texto más allá de la suma de sus componentes, poniendo énfasis en las formas 
en que éste se constituye y adquiere un sentido particular en el contexto en que se desarrolla, como unidad significante, más que la suma de frases (Esparza, 2006).

Respecto de la estrategia de análisis para este nivel, Stecher (2009) recomienda realizar un 'análisis lingüístico', el cual permitiría dar cuenta del modo como los distintos rasgos gramaticales, fonológicos, de vocabulario, de estructura global y cohesión, entre otros, producen efectos de sentido, es decir, impactos en las identidades, acciones y representaciones sociales. Lo fundamental de este tipo de análisis, entonces, se centra en dar cuenta de los sentidos que instancia esa organización textual, la trama de articulaciones diversas de la suma de componentes lingüísticos.

La utilización del análisis lingüístico permite reconocer los sentidos que el texto moviliza a partir de la compleja red de recursos que operan en su configuración. Con el fin de analizar esa construcción, es posible realizar una serie de entradas analíticas lingüísticas, a partir de sus aspectos fonéticos o fonológicos, o morfológicos, o sintácticos, o lexicológicos o lexicográficos, o semánticos, o bien a través de los actos de habla, entre otras posibilidades. Dentro de ese repertorio de alternativas, la investigación desde la que surge este artículo opta por poner atención en los aspectos cohesivos que configuran el texto, es decir, el modo en que los recursos lingüísticos se relacionan para dar un particular sentido y entendimiento dentro de una práctica social específica, o más bien, el modo en que se organiza el lenguaje y sus componentes para construir un texto que exprese lo que se desea comunicar, y a la vez sea entendido por los sujetos que forman parte de esa práctica.

Figura $N^{\circ} 2$. Síntesis $1^{\circ}$ Nivel: Discurso como Texto

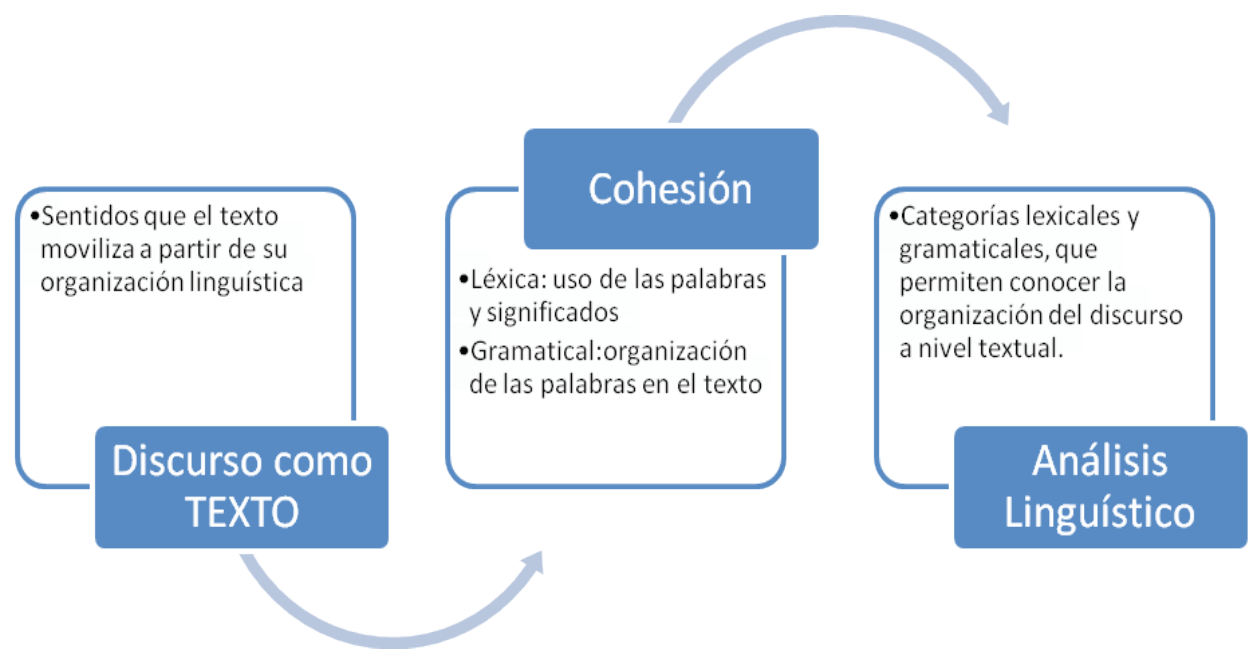

Fuente: elaboración propia.

De este modo, a través de la 'cohesión' y sus aspectos léxicos y gramaticales se realizará la primera entrada analítica, mediante la comprensión de los diversos procedimientos lexicales, que hacen referencia a las palabras y sus significados, y gramaticales, que hacen 
alusión al empleo de recursos morfosintácticos. En cada caso, se considera un conjunto de criterios que se incorpora a una Matriz de Análisis Lingüístico Lexical y Gramatical, donde se define cada uno de los criterios y las preguntas orientadoras para el análisis.

El segundo nivel del modelo tridimensional de Fairclough se sostiene sobre la base de que todo evento discursivo debe ser pensado en términos de los procesos de producción que le han dado lugar, así como de los procesos de recepción/interpretación a los que se ve sometido en la trama social. Estos procesos se caracterizan por su carácter dialéctico, en tanto se enfrentan y dialogan las convenciones discursivas con las intervenciones creativas en las diferentes instancias de comunicación, denominadas por Fairclough (2008) como aspectos semióticos, entendidos como variedades discursivas y formas de creación de significados de un orden social que organizan un orden del discurso, entendido como la manera en que las diferentes variedades discursivas y los diferentes tipos de discursos son ubicados juntos en la red. Es decir, el orden del discurso no es un sistema cerrado o rígido, sino más bien un sistema abierto que queda expuesto al peligro ${ }^{4}$ como consecuencia de lo que sucede en las interacciones efectivas (Fairclough, 2008).

En síntesis, este nivel centra su atención en los modos en que los sujetos producen e interpretan los textos a partir de un conjunto de recursos discursivos socialmente disponibles y relativamente estables que constituyen los órdenes del discurso, a partir de lo cual se reproducen las convenciones y se crean nuevas expresiones de discurso en los procesos de recepción e interpretación. A partir de esta concepción del discurso como práctica, se reconoce la existencia de múltiples discursos o voces en el discurso mayor, que de acuerdo con Julia Kristeva (1981), refieren a la intertextualidad, esto es, la propiedad de los discursos de ser un producto de la mezcla y la combinación de distintos recursos discursivos (géneros, textos, estilos) que operan como estabilizadores de la red u orden del discurso ${ }^{5}$.

De acuerdo con Fairclough (1992), la intertextualidad debe ser entendida de dos formas: la intertextualidad constitutiva o interdiscursividad, que representa los cambios o las modificaciones de los órdenes del discurso; y la intertextualidad manifiesta, que se caracteriza por la alternancia de diferentes textos o tipos de discurso en un texto, formas textuales donde los discursos están contenidos dentro de otro texto, y a su vez están mezcladas en forma compleja y difícil de distinguir y separar. En cuanto a los tipos de intertextualidad, en el estudio se opta por analizar la intertextualidad manifiesta, es decir, desentrañar los múltiples discursos que están presentes dentro del discurso curricular de la formación inicial docente y de género, abordando sus condiciones de producción a partir de la consideración de cuatro categorías: representación discursiva, presuposición, negación y metadiscurso.

Para el autor, existe un espacio de creatividad en el orden del discurso a partir de las reinterpretaciones de los sujetos en los contextos específicos y en las interacciones.

$5 \quad$ Si bien en este nivel se hace referencia a un 'orden del discurso', no se considera dentro del análisis, pues, de acuerdo con Fairclough (1992), eso forma parte de una propiedad de la intertextualidad constitutiva o interdiscursividad, que se verá en el tercer nivel. Este segundo nivel se preocupa por la intertextualidad manifiesta que identifica los elementos de ese orden. 
Figura $N^{\circ} 3$. Síntesis $2^{\circ}$ Nivel: Discurso como Práctica

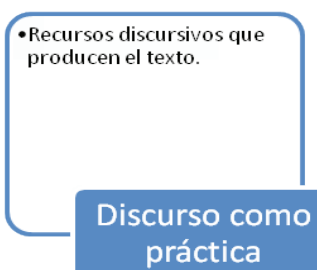
práctica

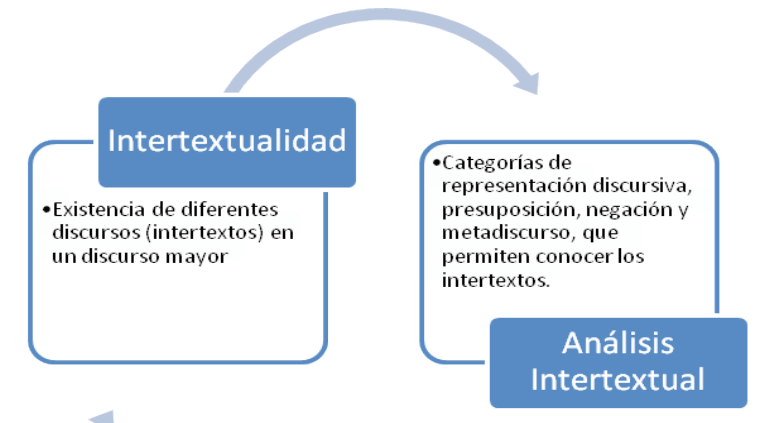

Fuente: elaboración propia.

Las categorías mencionadas -en conjunto con otras- han sido trabajadas por Burgueño (2006) en su estudio "Relaciones de intertextualidad en discursos políticos presidenciales en Chile". En este caso, he seleccionado las categorías que entregan información relevante a los propósitos del estudio.

Estas categorías se definen a partir de los aportes de Fairclough (1992), Marinkovich y Benítez (2004) y Burgueño (2006): la representación discursiva se refiere a la opción que toma el/la hablante frente a la forma de representar un relato discursivo, es decir, se opta por ser representado de una manera determinada y no de otra, considerando para ello el contexto en el que se desarrolla; la presuposición corresponde a los aspectos enunciados por el productor del texto como ya establecidos o dados, es decir, no se cuestionan, se asumen como ciertos; la negación no sólo incluye el uso explícito de negaciones, sino que también se emplea para aportar información relevante que las rectifiquen, lo que permite problematizar el discurso; finalmente, el metadiscurso hace alusión a la posición que toma el/la hablante sobre o fuera de su propio discurso, en términos de control o manipulación del mismo, a su vez, distingue diferentes niveles en su propio texto y se distancia con respecto a alguno de ellos.

El tercer nivel, de acuerdo a los planteamientos de Fairclough (2003, 1995, 1992, 1989), señala que todo evento discursivo debe ser pensado como parte de una práctica social. Como tal, se encuentra inserto en un conjunto de situaciones, instituciones y macro contextos que condicionan los usos del lenguaje. De esta forma, el texto está condicionado por la situación, la institución y la estructura social de la que es parte, y a la cual contribuye a partir del poder constitutivo de los sentidos que moviliza al producir el orden social, sosteniendo o socavando las relaciones de dominación en un particular dominio de la sociedad, dando espacio para su reproducción y creatividad.

Lo interesante de este tercer nivel de análisis es que considera los efectos políticos e ideológicos que se configuran dentro de esa práctica social específica ${ }^{6}$, los cuales operan, sostienen y transforman las relaciones de poder que existen en ese contexto. En este sentido, los referentes teóricos que fundamentan este posicionamiento nacen de las reflexiones de

\footnotetext{
6 En este caso se trata del diseño curricular de formación inicial docente de Pedagogía en Educación Básica, inserto en una institución y en un conjunto de prácticas político-educativas de carácter nacional y global.
} 
Gramsci sobre hegemonía cultural (Brocoli, 1979), en cuanto a la existencia de un control discursivo logrado a través de los procesos de estabilización de órdenes del discurso, a los que los sujetos acuden para actuar discursivamente en la vida social.

La lógica de este planteamiento sitúa la relación discursiva de los sujetos en función del uso de elementos de sentido común que se reproducen y naturalizan, entendiéndolos como formas de acción, representación e identificación no problematizadas, en la medida en que generan los efectos comunicativos deseados por convención. Ahora bien, dentro del ACD se considera que estos estabilizadores políticos e ideológicos del discurso forman parte de las estrategias de hegemonía cultural provisorias de un contexto sociocultural e histórico determinado.

En suma, este nivel del discurso como práctica social suma los aportes del primer y del segundo nivel, así como los respectivos análisis de tipo lingüístico e intertextual, para problematizar los usos lingüísticos y discursivos que allí operan, los cuales, en conjunto, generan órdenes del discurso como insumos para los/as hablantes, de modo que 'aparentemente' no se podrían usar otros discursos para comunicarse efectivamente. El análisis de este nivel busca problematizar esos órdenes, interrogándose por cómo los contextos socioculturales, institucionales e ideológicos específicos operan en la reproducción de esos discursos.

Para llevar a cabo un análisis desde esta concepción del discurso es relevante considerar la forma en que éste se construye y se condiciona por la 'situación, la institución y la estructura social' de la que es parte. Esto quiere decir que los discursos no se encuentran separados de la realidad donde han sido producidos, siendo las prácticas discursivas locales e institucionales decisivas en el levantamiento de los discursos específicos del campo en cuestión. A partir de este conjunto de ámbitos se construye un 'orden del discurso' que moviliza ciertos sentidos de orden social, constituidos sobre la base de relaciones de poder que originan significados incuestionables y prácticas de veracidad.

Es por ello que resulta pertinente considerar un 'análisis social explicativo' que, de acuerdo con Stecher (2009), busca dar cuenta de las condiciones sociocontextuales de los eventos discursivos en estudio, y cómo éstos participan en determinadas formas de producción, sostenimiento y transformación del discurso en ese contexto particular. El análisis de este nivel considera tres grandes ámbitos: determinaciones sociales, ideologías y efectos sociales.

Figura $N^{\circ}$ 4. Síntesis $3^{\circ}$ Nivel: Discurso como Práctica Social

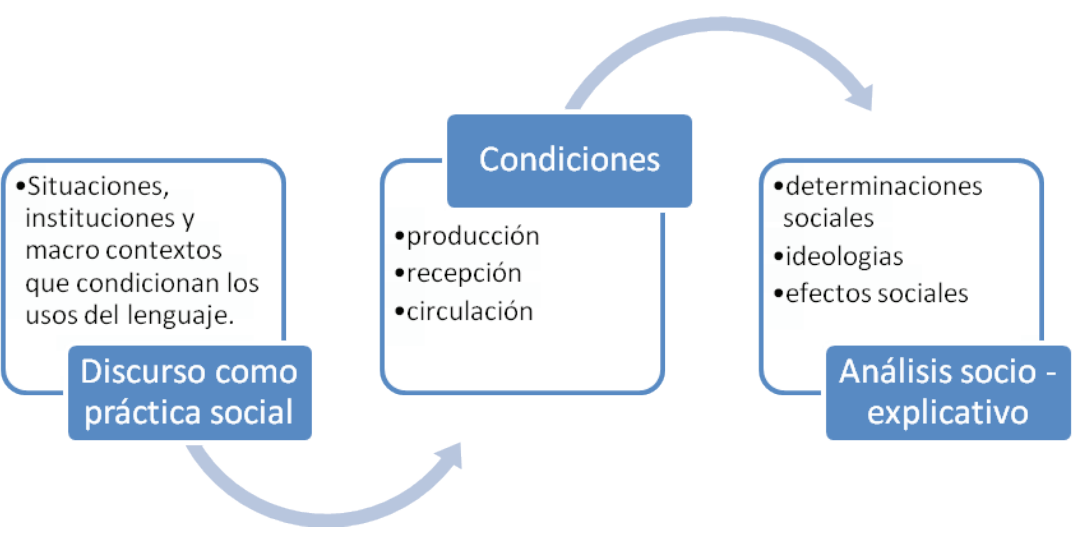

Fuente: elaboración propia. 
Las 'determinaciones sociales' aluden a las condiciones de producción de modelos de formación docente instalados, ante lo cual se pregunta: ¿cuáles son las condiciones institucionales y sociales de producción de los discursos curriculares? En segundo lugar, las 'ideologías' enfatizan las condiciones de recepción de estas creencias y su integración en el discurso curricular institucional, punto donde se pregunta: ¿qué ideologías priman en la formación de profesores y profesoras? Finalmente, los 'efectos sociales' hacen referencia a las condiciones de circulación de los discursos que generan impactos en la formación y en los discursos hegemónicos no problematizados, pudiendo interrogarse: ¿qué efectos políticos e ideológicos se configuran dentro de esta práctica social discursiva?

En concordancia con los propósitos de la investigación, y en función del interés de problematizar y comprender la realidad abriendo espacios a nuevas perspectivas de interpretación y explicación de los fenómenos sociales, se considera pertinente operar con información que profundice en los significados y en las formas de legitimación de los discursos curriculares de la formación inicial docente. De este modo, el corpus discursivo a analizar corresponde, por una parte, a una selección de documentos declarativos y curriculares propios del proceso de diseño curricular y, por otra parte, al discurso de los sujetos partícipes de esos procesos de diseño curricular en las instituciones formadoras.

En términos interpretativos, se propone realizar un recorrido sistemático del corpus y su contexto, mediante una exploración detallada e integrada a través de los niveles de análisis, es decir, cada nivel recoge lo señalado por el análisis anterior, hasta que finalmente el último nivel de práctica social contiene a todo el corpus en su análisis. En cuanto a los procedimientos de trabajo en el estudio, se considera para cada nivel la construcción de una 'matriz de análisis', a partir del tipo de análisis a realizar (lingüístico, intertextual y socio explicativo), sus criterios, categorías y preguntas orientadoras.

Tabla $N^{\circ} 1$. Niveles, tipos de análisis y selección del corpus

\begin{tabular}{|l|l|l|}
\hline \multicolumn{1}{|c|}{ Nivel } & Tipo de Análisis & \multicolumn{1}{c|}{ Selección del Corpus } \\
\hline Texto & Lingüístico & $\begin{array}{l}\text { - Entrevistas } \\
\text { - Documentos Curriculares: Principios Misión/Visión y Perfil de } \\
\text { Egreso }\end{array}$ \\
\hline Práctica & Intertextual & $\begin{array}{l}\text { - Entrevistas } \\
\text { - Documentos Declarativos y Curriculares: Principios Misión/Vi- } \\
\text { sión y Perfil de Egreso, Programas de Actividades Curriculares }\end{array}$ \\
\hline Práctica & Socio - Explicativo & $\begin{array}{l}\text { - Entrevistas } \\
\text { - Documentos Declarativos y Curriculares: Principios Misión/Vi- } \\
\text { sión y Perfil de Egreso, Programas de Actividades Curriculares, } \\
\text { Acuerdos de Acreditación, Modelos de Formación }\end{array}$ \\
\hline
\end{tabular}

Fuente: elaboración propia.

El posicionamiento de esta investigación señala que "tanto la teoría como la metodología son eclécticas, esto es, se incorporan las teorías y los métodos que resulten útiles para comprender y explicar el objeto sometido a investigación" (Wodak, 2003: 109). 
Por ende, en la selección de técnicas e instrumentos prima la posibilidad de obtener la información requerida en conformidad con los objetivos de investigación y la problemática tratada, procurando establecer un diálogo entre las diferentes técnicas e instrumentos y sus referentes epistemológicos.

\section{REFERENCIAS BIBLIOGRÁFICAS}

Broccoli, A. (1979). Antonio Gramsci y la educación como hegemonía. México D.F.: Editorial Nueva Imagen.

Butler, J. (1997). Mecanismos psíquicos del poder. Madrid: Ediciones Cátedra.

Burgueño, C. (2006). Relaciones de intertextualidad en discursos políticos presidenciales. Literatura y Lingüística, (17), 221-241.

Cherryholmes, C. (1999). Poder y crítica: Investigaciones posestructuralistas en educación. Barcelona: Pomares Corredor.

Denzin, N. (2008). Los nuevos diálogos sobre paradigmas y la investigación cualitativa. Un compromiso en la relación universidad - sociedad. Reencuentro, (52), 63-76. Recuperado el 15 de abril de 2012 desde http://www.redalyc.org/articulo.oa?id=34005206

. \& Lincoln, Y. (2005). Introduction. The Discipline and Practice of Qualitative Research. In N. Denzin \& Y. Lincoln (Eds.), The Sage Handbook of QualitativeResearch. $3^{\text {th }}$ Edition (pp. 1-19). Thousand Oaks: Sage Publications. Retrieved on April 15, 2012 from http://www.sagepub.com/upmdata/40425_Chapter1.pdf

Delgado, J. (1994). Métodos y técnicas cualitativas de investigación en ciencias sociales. Madrid: Editorial Síntesis Psicología.

Esparza, M. (2005). Pautas para el análisis de la cohesión y coherencia en textos españoles. RILCE. Revista de Filología Hispánica, vol. 22 (1), 59-89.

Fairclough, N. (2009). Políticamente correcto: La política de la lengua y la cultura. Discurso \& Sociedad, vol.3 (3), 495-512.

. (2008). El análisis crítico del discurso y la mercantilización del discurso público: Las universidades. Discurso \& Sociedad, vol.2 (1), 170-185.

(2003). El análisis crítico del discurso como método para la investigación en ciencias sociales. En R. Wodak y M. Meyer (Comps.), Métodos de Análisis Crítico del Discurso (pp. 179203). Barcelona: Gedisa.

. (1995). Critical Discourses Analysis. The Critical Study of Language. London/New York: Longman. Traducción y adaptación de Federico Navarro para la Cátedra de Lingüística General (Dr. Martín Menéndez) de la Facultad de Filosofía y Letras, Universidad de Buenos Aires, Argentina. Recuperado el 20 de noviembre de 2011 desde http://discurso.files.wordpress.com/2009/02/ fairclough1995analisis-critico-del-discursocap-1trad-navarro1.pdf

. (1992). Discourse and Social Change. Cambridge: Polity Press.

(1989). Language and Power. London: Longman.

Foucault, M. (2008). Gestionar los ilegalismos. En R. Droit, Entrevistas con Michael Foucault (pp. 47-57). Buenos Aires: Paidós.

. (1989). La arqueología del saber. México D.F.: Siglo XXI.

. (1970). El orden del discurso. Barcelona: Tusquets Editores.

Iñiguez, L. (2003). Análisis del discurso: Manual para las ciencias sociales. Barcelona: UOC.

Kristeva, J. (1981). Semiótica. $2^{\text {da }}$ Edición. Madrid: Editorial Fundamentos.

Marinkovich, J. y Benítez, R. (2004). Aproximaciones al análisis intertextual del discurso científico. Signos. Revista de Lingüística, vol.33 (48), 117-128.

Meyer, M. (2003). Entre la teoría, el método y la política: La ubicación de los enfoques 
relacionados con el ACD. En R. Wodak y M. Meyer (Comps.), Métodos de Análisis Crítico del Discurso (pp. 35-39). Barcelona: Gedisa.

Palacios, L. (2009). Epistemología y pedagogía de género: El referente masculino como modo de construcción y transmisión del conocimiento científico. Horizontes Educacionales, vol.14 (1), 6575. Recuperado el 15 de junio de 2010 desde http://helios.dci.ubiobio.cl/revistahorizontes/Revista/ files/revistas/2009

Stecher, A. (2009). El análisis crítico del discurso como herramienta de investigación psicosocial del mundo del trabajo. Discusiones desde América Latina. Universitas Psychologica, vol.9 (1), 93 107.

Van Dijk, T. (2009). Discurso y poder. Barcelona: Gedisa.

Wodak, R. (2003). De qué trata el análisis crítico del discurso (ACD). Resumen de su historia, sus conceptos fundamentales y su desarrollo. En R. Wodak y M. Meyer (Comps.), Métodos de Análisis Crítico del Discurso (pp. 17-34). Barcelona: Gedisa. 